\title{
Hue magnitude estimates as relative judgments
}

\author{
C. H. ELZINGA and CH. M. M. de WEERT \\ Psychologisch Laboratorium, University of Nijmegen, Nijmegen, Netherlands
}

\begin{abstract}
A simple model in terms of nonlinear opponent color codes for the veiling of a weak hue component by a strong one in mixtures of unique yellow and unique green is explored experimentally by means of a procedure which is an extension of the classical hue magnitude estimation procedures. The model is extended towards a more general model for the veiling phenomenon. An experimental validation of the procedure is included. Independent evidence for a hypothesis of Guth and Lodge (1973) regarding inhibition of the red/green opponent system by the yellow/blue opponent system was found when brightness estimates were conceived of as a function of the log luminance of the yellow stimulus component.
\end{abstract}

Opponent colors theories assume that the linear nonopponent output signals of three photopigments are recoded into three linear signals, namely a whiteness/blackness signal, a red/green signal, and a yellow/blue signal. To account for the Bezold-Brücke hue shift and Abney's hue shift, it is furthermore assumed that these opponent linear signals are recoded into at least three nonlinear signals. For an excellent substantial and historical review of opponent colors theory, the reader is referred to Hurvich (1977).

Krantz (1975a, 1975b) first provided a formal basis for opponent colors theory which is at the same time a measurement theory for the cancellation-energy measurements of Hurvich and Jameson (1957; James \& Hurvich, 1955). Within the set of all lights, $A$, Krantz distinguishes two subsets, $A_{1}$ and $\mathbf{A}_{2}$. The set $\mathbf{A}_{1}$ consists of all lights that are neither reddish nor greenish; $A_{2}$ consists of all lights that are neither bluish nor yellowish. Consequently, the set $\mathbf{A}_{1} \cap \mathbf{A}_{2}$ consists of all lights that appear colorless. All lights $a \in \mathbf{A}_{1} \cup \mathbf{A}_{2}$ are called equilibrium lights or unique colors. If $a \in \mathrm{A}_{1}, a$ is called a unique yellow or blue; if $a \in \mathrm{A}_{2}, a$ is called a unique red or green.

If the axioms of Kranz' theory are satisfied, A can be represented by a convex cone, $\mathrm{C}$, in a linear three-dimensional space, i.e., there exists a homomorphism, $\Phi$, which maps each light $a \in A$ onto a vector $\Phi(a)=\left[\phi_{1}(a), \phi_{2}(a), \phi_{3}(a)\right] \in C$. The functions (coordinates of $C$ ) $\phi_{1}$ and $\phi_{2}$ represent the linear form of the red/green and yellow/blue opponent cancellation codes; i.e., the functions $\phi_{1}(i=1,2)$ are linear with respect to color mixture operations:

The authors' address is Department of Mathematical Psychology, Psychologisch Laboratorium, University of Nijmegen, Erasmuslaan 16, Nijmegen, Netherlands.

$$
\phi_{\mathrm{i}}[(\mathrm{u} * a) \circ(\mathrm{v} * b)]=\mathrm{u} \cdot \phi_{\mathrm{i}}(a)+\mathrm{v} \cdot \phi_{\mathrm{i}}(b)
$$

for all, $a, b \in \mathrm{A}$ and all $\mathrm{u}, \mathrm{v} \in \mathrm{R}^{+}$. Here * and $\mathrm{O}$ denote, respectively, an overall change in energy level and additive mixture of colored lights. In fact, the connotation of Equation 1 is that $\Phi$ maps the cone $\langle\mathrm{A}, \mathrm{O}, *\rangle$ onto the cone $\left\langle\mathrm{R}^{+},+, \cdot\right\rangle$.

An important property of the $\phi_{i}$ is that $\phi_{i}(a)=$ $0 \Leftrightarrow a \in \mathrm{A}_{\mathrm{i}}$ for $\mathrm{i}=1,2$. From the viewpoint of empirical validity of Krantz' axiomatization, of interest are those axioms which imply closure of the $A_{i}$ under the operations $O$ and *. These axioms have been tested extensively, under dark adaptation, by Larimer, Krantz, and Cicerone (1974, 1975). Krantz' axioms are independent from the state of adaptation of the eye in the sense that closure of the $A_{i}$ should hold for any state of adaptation. However, the makeup of the $A_{i}$ may, and in fact does, change with changes of the state of adaptation. Cicerone, Krantz, and Larimer (1975) tested for closure of the $A_{i}$ under different conditions of color adaptation.

Krantz' axioms are a set of axioms which describe a state of affairs in a physical space; now the psychophysical problem is to establish the relation between the opponent linear cancellation codes, $\phi_{i}$, and the apparently nonlinear codes, $f_{i}$, which occur later on in the visual system and which are related to subjective color experience. This problem could also be formulated as the problem of establishing functions, $F_{i}$, that map the $\phi_{i}$ onto codes for subjective color experience, $f_{j}$. These transducer functions, $F_{i}$, cannot be one-argument functions because of what Krantz has called the veiling phenomenon.

Veiling is the masking of a weak hue component by a strong one. Imagine a unique green light, $a$, and a unique yellow, $b$. According to Krantz' axioms, the red/green cancellation code for $b$ must equal 
zero because $b \in \mathrm{A}_{1}: \phi_{1}(b)=0$; and because $a \in \mathrm{A}_{2}$, we also have $\phi_{2}(a)=0$. But because both lights are colored, we must have $\phi_{2}(b)>0$ and $\phi_{1}(a)>0$. Now consider the mixture $a \circ b$ : because $\phi_{1}$ must satisfy Equation 1, we have, with $\phi_{1}(b)=0, \phi_{1}(a \circ b)=$ $\phi_{1}(a)+\phi_{1}(b)=\phi_{1}(a)$.

Thus the red/green cancellation code $\phi_{1}$ has the same numerical value for $a$ and the additive mixture $(a \circ b)$. But direct magnitude estimation of the greenness of $a$ and (a $O b$ ) would reveal that the subjective impression of greenness evoked by $(a \circ b)$ is considerably less than that evoked by $a$ alone. Raising the physical intensity of $b$ in the mixture would not alter the red/green cancellation value of the mixture, but would cause the subjective impression of greenness from that mixture to vanish almost completely. Thus the addition of yellow to green seems to veil the subjective impression of greenness, and thus the veiling phenomenon seems to rule out any expression of the form $f_{i}=F_{i}\left(\phi_{i}\right)$. The existence of a veiling phenomenon was already suggested by Yager and Taylor (1970) when their empirical hue coefficient function grew faster than was predicted on the basis of their estimated luminance thresholds for perceived yellowness and greenness and the estimates of the exponents when hue magnitude estimates (HMEs) were expressed as power functions of the luminance. However, their estimates of these exponents were about four times smaller than the exponents estimated by Raaijmakers and de Weert (1975). The latter conducted an experiment in which they obtained HMEs of greenness for different values of the luminance of a unique green stimulus under admixture of two levels of a unique yellow. The HMEs clearly seemed to show veiling of the green by the admixed yellow. However, by describing their data as a function of the proportion of the luminance of the green in the mixture, it seemed as if no veiling had occurred at all. They concluded that their subjects probably had not been able to give absolute judgments about the amounts of green in the stimuli. Raaijmakers and de Weert suggested that the relative strength of the chromatic and achromatic signals might be the only measures available to the subject, so that the HMEs produced by their subjects could not be regarded as absolute judgment about the nonlinear opponent code for green. A further indication that this might be true is the fact that the logarithm of the power functions as obtained by Yager and Taylor grew faster at lower luminance levels and only became linear with log-luminance at higher luminance levels. If the suggestion of Raaijmakers and de Weert, that only information about the relative strength of chromatic and achromatic signals is available to the subject, a rather simple model for hue magnitude estimates in terms of the nonlinear opponent color codes $\mathrm{f}_{\mathrm{i}}$ is given by

$$
H_{i}=c B\left(\frac{f_{1}}{f_{1}+f_{2}}\right) .
$$

Here $\mathrm{H}_{i}$ denotes the hue magnitude estimate associated with $f_{i}$, and $c B$ is a positive scalar transformation of the brightness estimate, $B$. We shall assume that the $f_{i}$ are strictly monotone with luminance and thus with the $\phi_{i}$. With this very weak assumption, it is easy to see that this model recognizes veiling: if, given a constant $\phi_{1}$ level of a mixture, $\phi_{2}$ increases, $f_{2}$ will also increase, thus diminishing the ratio $f_{1} /\left(f_{1}+f_{2}\right)$, and, given a suitable choice of $c, H_{1}$ will decrease. This corresponds to the veiling of greenness caused by raising the intensity of a unique yellow in a mixture of unique yellow and unique green. For a constant $\phi_{2}$ level, $\mathrm{H}_{1}$ will be monotone with $\phi_{1}$.

Notice that Equation 2 assigns special status to brightness as a psychologically more primitive aspect of light than hue. However, this is not meant to say that the brightness sensation arises independently from the $f_{i}$. If we denote the nonlinear opponent code for whiteness/blackness as $f_{3}$, brightness could be thought of as a function of all three nonlinear opponent codes: $B=h\left(f_{1}, f_{2}, f_{3}\right)$. Such a proposal has already been made by Guth, Donley, and Marocco (1969).

The experiment to be reported here concerns the evaluation of Equation 2 as a candidate for explaining the veiling phenomenon without accepting the assumption that the nonlinear opponent hue codes are multiargument functions of the linear opponent codes.

\section{METHOD}

\section{Apparatus}

In Figure 1, a diagram of the equipment is given.

Two xenon arc lamps were used as light sources. The light of these sources was transformed into monochromatic light with the help of two Carl-Leiss prism monochromators. Monochromator M1 provided the yellow beam, monochromator $M 2$ the green one. The intensity of the sources was controlled by varying circular neutral density wedges $\mathrm{W} 1$ and $\mathrm{W} 2$. Through beamsplitter BS, the yellow and the green beam were mixed. With the help of lens ML, the target was seen in Maxwellian view and subtended $1.5^{\circ}$. The various mixtures of the luminances of the yellow and the green beam were selected by choosing the appropriate neutral density filters from $\mathrm{Wr} 1$ and $\mathrm{Wr} 2$. Wr1 and $\mathrm{Wr} 2$ both consisted of a disk in which a number of different density filters were placed. By rotating the disks, different filter combinations were chosen. $S$ is an electromagnetically driven shutter with a circular diameter of $5 \mathrm{~cm}$. A source, $\mathrm{C}$, could be projected onto a flickervane, $F$, for the purpose of equating the yellow and the green beam for luminance. $\mathrm{C}$ was also used for a constant and faint illumination of the surroundings of the target. The brightness level of the target surroundings was kept constant during the whole experiment and was much lower than that of the least bright stimulus.

\section{Subjects}

Three subjects, A, C, and F, served in the experiment. None 


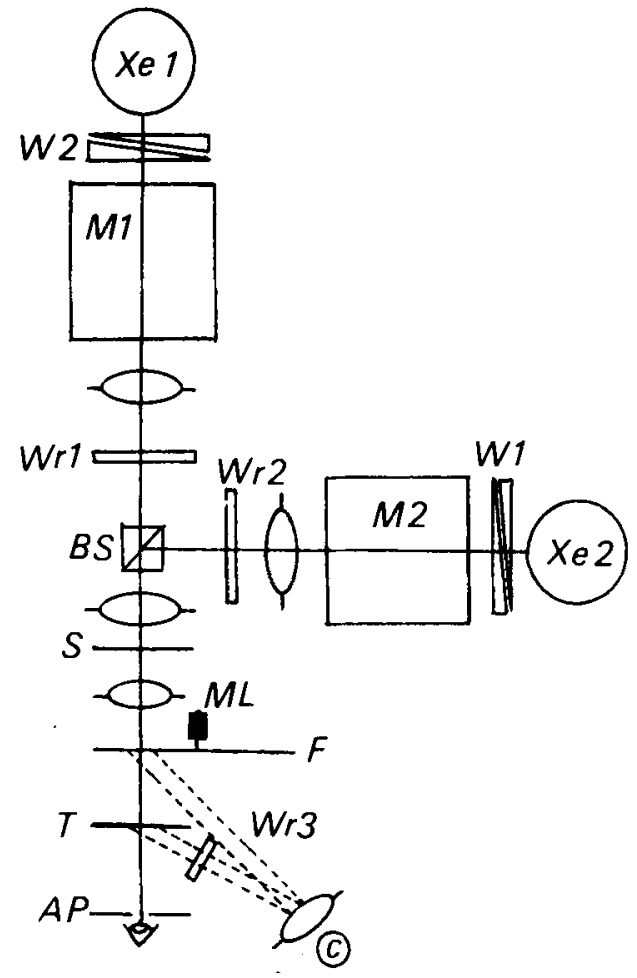

Figure 1. Diagram of the optical equipment. Monochromators $M 1$ and $M 2$ are fed by 150-W xenon arc lamps Xel and Xe2. Wr1 and $W_{r} 2$, neutral density filters; $W 1$ and $W 2$, circular neutral density wedges; BS, beamsplitter; $S$, electromagnetically driven shutter; $F$, flickervane; $T$, test target, subtending $1.5^{\circ} ; W_{\mathbf{R 3}}$, neutral density filter; $A P, 2-m m$ circular artificial pupil; $C$, calibration light source.

of them had had any prior experience with color experiments. All subjects had normal color vision. The subject used his right eye; the left eye was kept covered during the whole experimental session. The head of the subject was fixed by using a chin- and foreheadrest.

\section{Determination of Unique Colors}

After a dark-adaptation period of $10 \mathrm{~min}$, a rough determination of the wavelengths of the unique colors was made. Following a readaptation period of $3 \mathrm{~min}$, seven different wavelengths around the estimated unique wavelengths were presented in a random-block design. Each wavelength was presented 10 times. The subjects had to say whether red or green was present in the case of yellowish stimuli and, in the case of greenish stimuli, whether yellow or blue was seen. The $50 \%-50 \%$ value was taken as the wavelength of the unique color. In Table 1 , the wavelength values, at a retinal illumination level of 168 photopic trolands, of the unique colors of each subject are presented.

\section{Procedure}

After determination of the wavelength of the unique colors as described above, the luminances of both colors were equated according to the flickerphotometric procedure at the 0.0 values of the density filters. Subsequently, the subject was instructed about his task as follows: "Each 30 seconds, after a click is heard, a short flash will be given, the color of which varies between yellow and green." The range of colors was then shown to the subject. "Your task is to assign a number to the brightness of these flashes and to assign numbers to the amount of green and the amount of yellow present in the flash. These judgments should feel right. You may use whole numbers, fractions, or a combination of the two. Try to make the numbers directly proportional to your impression of the brightness, the amount of green and the amount of yellow present in the flash. So, for each flash, you have to judge three different aspects of the flash: the brightness, the amount of yellow, and the amount of green. Assigning a number to the brightness, you should try to ignore the greenness and the yellowness of the flash; in assigning a number to the amount of green (yellow), you should try to ignore the brightness and the amount of yellow (green) in the flash. Constantly try to remember that the number you use should be proportional to your own subjective impression of the brightness and the amounts of each hue present in the flash. All flashes are mixtures of yellow and green, so you should avoid the use of the number zero. Try not to worry about being consistent, small variations are quite normai for this kind of task." Thereafter, the subject was asked to restate the instruction in order to test his comprehension of the task.

The stimuli were presented in random order, and the order of the judgments per presentation was also randomized. Each stimulus was presented five times. Stimuli were presented for $4 \mathrm{sec}$. Five hundred milliseconds before presentation, one 20 -msec click was presented as a warning signal.

From the first 15 presentations, a single stimulus was randomly selected. This stimulus was presented after each 15 th presentation, and the subject was then informed about the brightness and hue magnitude estimates he had produced at the first presentation of this stimulus.

Range of yellow and green luminance. In Table 2, six different levels of luminances of the yellow and the green are presented. The 36 combinations of these levels were randomly presented, each combination five times.

\section{Validation of the Experimental Method}

In fact, our experiment consisted of three different experiments as three different types of judgments were required from the subjects. One would expect that, if this procedure indeed corresponds to three separate experiments, hue magnitude estimates and brightness estimates from separate experiments should be scalar transformations of the corresponding estimates, as obtained in the combined experiments. Subject $F$ was used to test this hypothesis. Subject $F$ served in three separate experiments which were an exact replication of the experiment described above, except for the fact that in each of these experiments only one of the three kinds of estimates was required from the subject. The replications were run on 3 separate days in succession and 4 days after the original experiment with subject $F$ had taken place. To evaluate our prediction that the estimates from the original experiment were scalar transformations of the brightness and hue magnitude estimates obtained from the replications, we tried to predict the results of the combined experiments from the results of the replications

Table 1

Wavelength Settings for Unique Yellow and Unique Green

\begin{tabular}{ccc}
\hline Subject & Yellow (NM) & Green (NM) \\
\hline A & 586 & 513 \\
C & 581 & 520 \\
F & 574 & 516 \\
\hline
\end{tabular}

Table 2

Luminance Values of the Stimulus Components (in Trolands)

\begin{tabular}{lllllll}
\hline Green & 1.5 & 3.5 & 8.5 & 21 & 53 & 168 \\
Yellow & 1.5 & 3.5 & 8.5 & 21 & 53 & 168 \\
\hline
\end{tabular}


according to the model $Y=\beta X+\varepsilon$. As a measure of the goodness of fit of this model, we use a coefficient of determination: (d) which is a direct analogue of the squared correlation coefficient $\left(r^{2}\right)$. $d$ was used instead of $r^{2}$ because what matters is proportionality between $\mathrm{X}$ and $\mathrm{Y}$ and not primarily linearity. In Table 3, the coefficients of determination for the three types of judgments, together with the squared correlation coefficients and the estimated scalars $(\hat{\beta})$, are presented.

As can be seen from Table 3, the fit of the data to the hypotheses of scalar transformations is rather good, although the fit to the hypotheses of linear transformations is slightly better. The better fit of a linear model might be explained by assuming time-dependent response criteria. The figures in Table 3 seem to justify the conclusion that our method leads to the same kind of judgments as those which would have been obtained by running a separate experiment for each type of judgment. If this conclusion is correct, indeed, then our procedure has led to a considerable decrease in experimental effort as compared to conventional procedures of magnitude estimation in which only one type of judgment is required from the subjects.

\section{Theory of Conjoint Measurement}

The theory of conjoint measurement concerns the way in which independent variables determine a joint effect according to one or another rule of composition of independent variables. Here we shall be concerned only with those aspects of the theory which are directly relevant to the subject matter of this article. The interested reader is referred to Krantz, Luce, Suppes, and Tversky (1971) for a detailed and complete treatment of conjoint measurement structures.

Let $G$ and $Y$ denote, respectively, the set of all unique green lights and the set of all yellow lights, and let $\mathbf{M}$ denote the Cartesian product matrix of $\mathrm{G} \times \mathrm{Y}$, of which the elements $[\mathrm{m}(a, w)]$ are numbers representing the joint effect of the additive mixture of $a \in \mathrm{G}$ and $w \in \mathrm{Y}$, i.e., $\mathrm{G} \times \mathrm{Y}$ is weakly ordered and $\mathrm{m}$ is an ordinal scale on $\mathrm{G} \times \mathrm{Y}$.

$M$ satisfies monotonicity (or independence) whenever, for $a, b \in \mathrm{G}$ and some $w \in \mathrm{Y}, \mathrm{m}(a, w) \geqslant \mathrm{m}(b, w)$ implies $\mathrm{m}(a, x) \geqslant$ $\mathrm{m}(b, x)$ for all $x \in \mathrm{Y}$ and, for $x, y \in \mathrm{Y}$ and some $c \in \mathrm{G}, \mathrm{m}(c, x) \geqslant$ $\mathrm{m}(c, y)$ implies $\mathrm{m}(d, x) \geqslant \mathrm{m}(d, y)$ for all $d \in \mathrm{G}$. If $\mathrm{M}$ is an $\mathrm{n} \times \mathrm{n}$ matrix, there are $2\left(\frac{n}{2}\right)(n-1)$ possible tests of monotonicity. For all practical purposes, the above mentioned definition implies that a matrix $M$ satisfies monotonicity if there exists a permutation of rows and columns of $M$ such that within each row the elements

Table 3

Values of the Coefficient of Determination (d), the Squared Correlation Coefficients $\left(r^{2}\right)$, and the Estimated Scalars $(\hat{\beta})$ Resulting from the Prediction of Magnitude Estimates Obtained from the Combined Experiments Out of Magnitude Estimates of Three Separate Experiments

\begin{tabular}{lllr}
\hline \multicolumn{1}{c}{ Judgment } & $\mathrm{d}$ & $\mathrm{r}^{2}$ & \multicolumn{1}{c}{$\dot{\beta}$} \\
\hline Brightness & .82 & .90 & 1.19 \\
Amount of Yellow & .73 & .74 & .82 \\
Amount of Green & .80 & .83 & 1.05 \\
\hline
\end{tabular}

Table 4

Coefficients of Determination, Squared Correlation Coefficients, and Estimated Scalars Resulting from the Prediction of Brightness Estimates from the Sum of the Hue Magnitude Estimates

\begin{tabular}{cccc}
\hline Subject & $\mathrm{d}$ & $\mathrm{r}^{2}$ & $\overline{\mathrm{c}}$ \\
\hline $\mathrm{A}$ & .87 & .93 & .89 \\
$\mathrm{C}$ & .95 & .95 & .90 \\
\hline
\end{tabular}
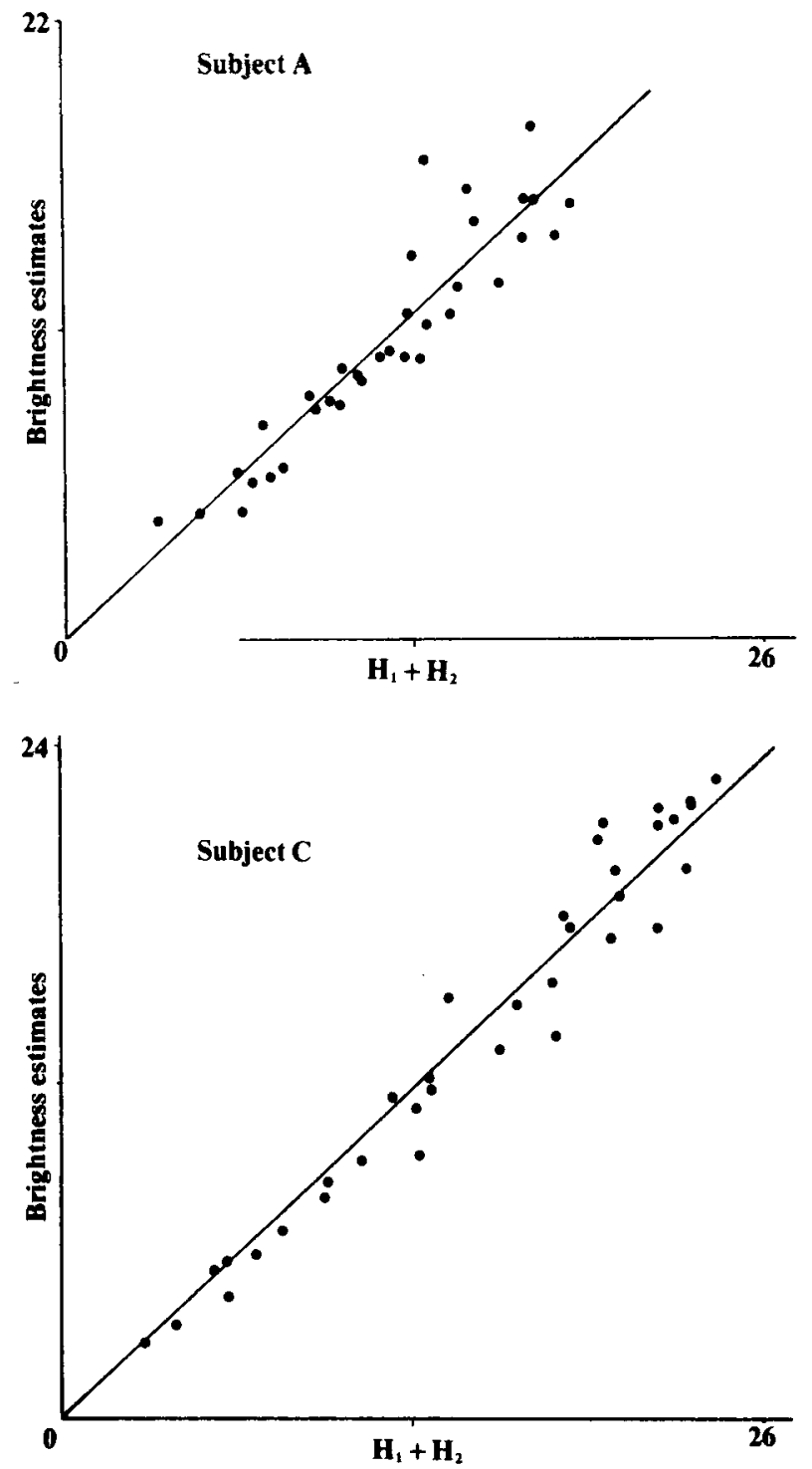

Figure 2. Brightness estimates vs. the sum of the hue magnitude estimates. Points represent the geometrical means of five presentations.

are nondecreasing from the left to the right and such that, within each column, the elements are nondecreasing from top to bottom.

$M$ satisfies double cancellation whenever, for all $a, b, c \in \mathrm{G}$ and all $w, x, y \in \mathrm{Y}, \mathrm{m}(a, x) \geqslant \mathrm{m}(b, w)$ and $\mathrm{m}(b, y) \geqslant \mathrm{m}(c, x)$ together imply $\mathrm{m}(a, y) \geqslant \mathrm{m}(c, w)$. If $\mathrm{M}$ is an $\mathrm{n} \times \mathrm{n}$ matrix there are $\left(\frac{n}{3}\right)^{2}$ triples to be checked with respect to double cancellation. These tests are not independent, since the conclusion of one may be one of the antecedent of another. For some of the triples, a test for double cancellation may not be possible because, for some triples, the antecedents may not allow for the conclusion of the double-cancellation rule. If $\mathbf{M}$ does not satisfy double cancellation, the violations may be one of two possible kinds of violations. A "strong" rejection occurs if both antecedents are strict inequalities and the conclusion doesn't hold. If, however, one of the antecedents is an equality and the conclusion doesn't hold, there is a violation also. This type of rejection is called "weak," because with finer grained data the equality antecedent might go either way, and in one of these cases the antecedents will not be apt to allow for a test of double cancellation. 
Table 5

Results of the Tests for Monotonicity and Double Cancellation of the Matrices of Ranks of $\log \left(\mathrm{H}_{\mathfrak{i}} / \mathrm{H}_{j}\right)$ for the Complete Set of Stimuli

\begin{tabular}{lrr}
\hline & \multicolumn{2}{c}{6 by 6 Matrix } \\
\cline { 2 - 3 } \multicolumn{1}{c}{ Cancellation Test } & A & C \\
\hline Acceptance & 399 & 400 \\
Weak Rejection & 0 & 0 \\
Strong Rejection & 1 & 0 \\
No Test Possible & 0 & 0 \\
Number of violations of monotonicity & 11 & 9 \\
\hline
\end{tabular}

Note-Total number of attempts of double-cancellation tests is $\left(\begin{array}{l}6 \\ 3\end{array}\right)^{2}=400$. Total number of tests of monotonicity is $\left(\begin{array}{l}6 \\ 2\end{array}\right) 10=150$.

Table 6

Results of the Tests for Monotonicity and Double Cancellation of the Matrices of Ranks of $\log \left(\mathrm{H}_{\mathbf{i}} / \mathrm{H}_{\mathbf{j}}\right)$ for the Reduced Set of Stimuli

\begin{tabular}{lcr}
\hline & \multicolumn{2}{c}{5 by 5 Matrix } \\
\cline { 2 - 3 } \multicolumn{1}{c}{ Cancellation Test } & A & C \\
\hline Acceptance & 100 & 100 \\
Weak Rejection & 0 & 0 \\
Strong Rejection & 0 & 0 \\
No Test Possible & 0 & 0 \\
Number of violations of monotonicity & 4 & 0 \\
\hline
\end{tabular}

Note-Total number of attempts of double-cancellation tests is $\left(\frac{s}{3}\right)^{2}=100$. Total number of tests of monotonicity is $\left(\begin{array}{l}5 \\ 2\end{array}\right) 8=80$.

If $M$ satisfies, apart from a number of axioms of which the empirical consequences are hard to evaluate, monotonicity and double cancellation, it can be proven, as shown by Krantz et al. (1971), that real-valued functions $\psi_{1}, \psi_{2}$ exist such that $\mathrm{m}(a, w)=$ $\psi_{1}(a)+\psi_{2}(w)$. The functions $\psi_{1}$ and $\psi_{2}$ are unique up to positive linear transformations with a common unit. There are several, rather laborious, methods for the construction of standard sequences that provide solutions for $\psi_{1}$ and $\psi_{2}$. For a discussion of some of these methods and the application of one of them, the reader is referred to Levelt, Riemersma, and Bunt (1972). Alternatively, a suitable nonmetric multidimensional scaling algorithm may be employed to solve for the scales $\psi_{1}$ and $\psi_{2}$.

\section{Results}

The most direct test of Equation 2 is to test the hypotheses that $\mathrm{H}_{\mathrm{i}}+\mathrm{H}_{\mathrm{j}}=\mathrm{cB}$ (c denotes a positive constant). The results of this test appear in Table 4 and Figure 2. In Table 4 the ds refer to the prediction of the brightness estimates from the sum of the HMEs. The lines in Figure 2 represent the predicted brightness estimates.

Clearly, these data support our hypotheses.

Equation 2 also leads to

$$
\log \left(H_{i} / H_{j}\right)=\psi_{i}\left(\log f_{i}\right)-\psi_{j}\left(\log f_{j}\right),
$$

which can be considered as a subtractive conjoint measurement model. If a subtractive representation $\ldots \log \left(\mathrm{H}_{\mathrm{i}} / \mathrm{H}_{\mathrm{j}}\right)$ exists, the matrix of ranks of $\log \left(\mathrm{H}_{\mathrm{i}} / \mathrm{H}_{\mathrm{j}}\right)$ should at least satisfy monotonicity and the doublecancellation rule. These properties were tested for both subjects $A$ and $C$. The results of these tests appear in Table 5. As can be seen from Table 5, the matrices of ranks of $\log \left(\mathrm{H}_{\mathrm{i}} / \mathrm{H}_{\mathrm{j}}\right)$ of both subjects do not satisfy monotonicity, nor does the matrix of subject A satisfy double cancellation.
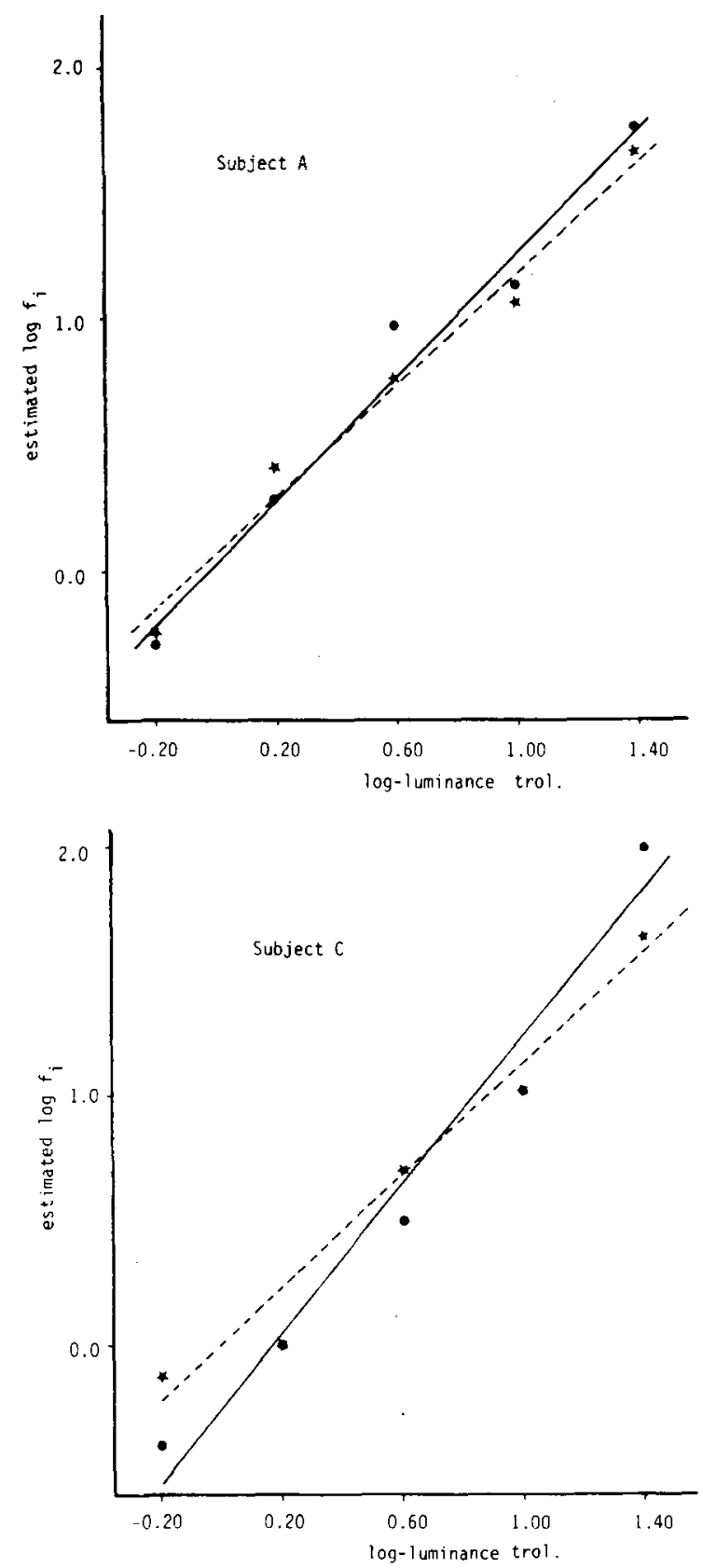

Figure 3. Estimated $\log f_{1}$ values vs. the $\log$ luminance of the stimulus components. The points represent the estimated $\log f_{1}$ values, the stars represent the estimated $\log f_{2}$ values. 
Table 7

Values of the Estimated Exponents $\left(\hat{\beta}_{\mathfrak{j}}\right)$ When Opponent Nonlinear Codes $f_{i}$ are Conceived of as Power Functions of the Luminance of the Stimulus Components

\begin{tabular}{ccccc}
\hline Subject & $\beta_{1}$ & $\mathrm{r}_{1}^{2}$ & $\beta_{2}$ & $\mathrm{r}_{2}^{2}$ \\
\hline $\mathrm{A}$ & 1.12 & .98 & 1.23 & .97 \\
$\mathrm{C}$ & 1.13 & .98 & 1.48 & .98 \\
\hline
\end{tabular}

However, in almost all the violations, the first row and column of these matrices are involved. No permutation of rows and columns leads to a substantial improvement of monotonicity without at the same time producing an increase in the number of violations of double cancellation. As the first row and column in these matrices refer to stimuli in which the physical amounts of yellow and/or green were highest, the violations of the necessary conditions of monotonicity and double cancellation could be considered as a response bias, caused by a restriction of the response range. It could also be true that Equation 2 is simply false for higher luminance levels. In any case, the next question is whether a more restricted set of stimuli could lead to a subtractive representation. We therefore computed the matrices of ranks of $\log \left(\mathrm{H}_{\mathrm{i}} / \mathrm{H}_{\mathrm{j}}\right)$, ignoring those stimuli in which the physical amounts of yellow and/or green were maximal. From the figures in Table 6, it is clear that these matrices turn out to satisfy monotonicity (almost) perfectly.

Now the conclusion seems justified that an acceptable solution exists for a subtractive representation for the reduced set of stimuli. Such a solution was obtained by using an algorithm which was developed by Roskam (Note 1) and can be considered as an extension of nonmetric multidimensional scaling algorithms such as Kruskal's.

If a subtractive representation for $\log \left(\mathrm{H}_{\mathrm{i}} / \mathrm{H}_{\mathrm{j}}\right)$ exists, scales $\psi_{i}\left(\log f_{i}\right)$ can be found which are unique up to positive linear transformations with a common unit. Conjoint analysis of the ranks of $\log \left(\mathrm{H}_{\mathrm{i}} / \mathrm{H}_{\mathrm{j}}\right)$ thus produces scales for $f_{1}$ and $f_{2}$ of the form

$$
\log f_{i}=\left[\psi_{i}\left(\log f_{i}\right)-\delta_{i}\right] / \alpha .
$$

As we are interested in the $f_{i}$, the additive constants $\delta_{\mathrm{i}}$ have to be estimated ( $\alpha$ can arbitrarily be chosen to equal 1). According to Equation 2, it must be true that $\mathbf{H}_{\mathrm{j}} / \mathrm{H}_{\mathrm{j}}=\mathrm{f}_{\mathrm{i}} / \mathrm{f}_{\mathrm{j}}$, so the parameters $\delta_{\mathrm{i}}$ and $\delta_{j}$ can be estimated by minimizing the function $\xi$ :

$$
\xi=\sum_{\mathrm{i}} \sum_{\mathrm{j}}\left[\log \left(\mathrm{H}_{\mathrm{i}} / \mathrm{H}_{\mathrm{j}}\right)-\left(\psi_{\mathrm{i}}-\delta_{\mathrm{i}}-\psi_{\mathrm{j}}+\delta_{\mathrm{j}}\right)\right]^{2} .
$$

The least squares estimates of the $\delta_{i}$ were obtained by using an algorithm developed by James and Roos (Note 2).
Having obtained these parameter estimates, estimates of the $\log f_{i}$ are easily obtained by subtracting the estimated constants from the corresponding scales $\psi_{i}$. In Figure 3, these estimates are plotted against the logarithm of the luminance of the stimulus components for both subjects.

Denoting the luminances of the stimulus components by $\mathrm{L}_{\mathrm{i}}$ and assuming that

$$
f_{i}=k_{i} L_{i}^{\beta_{i}}
$$

the coefficients $\beta_{\mathrm{i}}$ can be estimated by simple linear regression. These estimates are presented in Table 7 for both subjects, together with the squared correlation coefficients.

Furthermore, if Equation 2 is correct, the HMEs should be predictable from the estimated $\mathrm{f}$ scales and the observed brightness estimates by

$$
\mathrm{H}_{\mathrm{i}}=\mathrm{cB}\left(\frac{\hat{\mathrm{f}}_{\mathrm{i}}}{\hat{\mathrm{f}}_{1}+\hat{\mathrm{f}}_{2}}\right) \text {. }
$$

Here $\hat{f}_{i}$ denote the estimated opponent nonlinear codes. In Figure 4, plots of the predicted HMEs against the observed HMEs are given for both subjects. In Table 8, the coefficients of determination and the squared correlation coefficients associated with these predictions are presented.

The fact that these squared correlation coefficients are somewhat higher than the coefficients of determination might be explained by assuming that, at the lowest luminance levels, subjects restrict the lower bound of their response range to the number 1 and are not willing or able to use fractions smaller than one. The plots in Figure 4 do suggest such a response effect.

\section{DISCUSSION}

On the basis of the aforementioned results, it seems safe to conclude that our model for hue magnitude estimates has not been refuted by out data. This indicates that the veiling phenomenon should be considered as the result of the response generating

Table 8

Coefficients of Determination (d) and Squared Correlation Coefficients $\left(r^{2}\right)$ Resulting from the Prediction of the Observed Hue Magnitude Estimates from the Estimated Scales for the Opponent Nonlinear Codes

\begin{tabular}{cccccc} 
& \multicolumn{2}{c}{ Yellow } & & \multicolumn{2}{c}{ Green } \\
\cline { 2 - 3 } \cline { 5 - 6 } Subject & $\mathrm{d}$ & $\mathrm{r}^{2}$ & & $\mathrm{~d}$ & $\mathrm{r}^{2}$ \\
\hline $\mathrm{A}$ & .92 & .98 & & .95 & .98 \\
$\mathrm{C}$ & .94 & .98 & & .96 & .98 \\
\hline
\end{tabular}



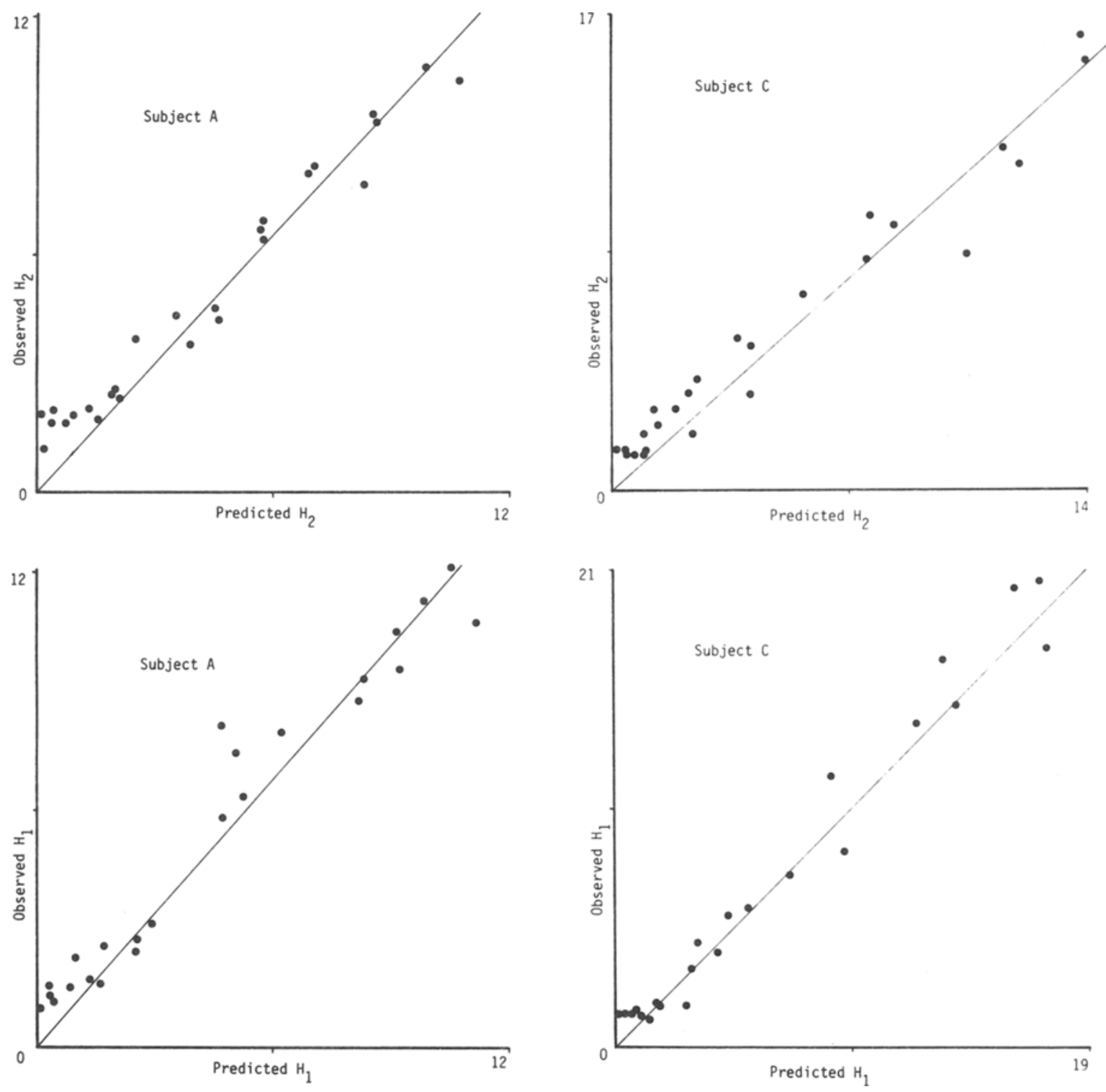

Figure 4. Observed magnitude estimates vs. magnitude estimates as predicted from the estimated $f_{i}$ values and the brightness estimates.

process and not of the signal transducing mechanism. Interesting as this may be, the model, as such, in no way can be considered as a complete account of the veiling phenomenon, because, as reported by Hurvich and Jameson (1951), broad-band light is judged white (colorless) if the luminance is raised sufficiently. This phenomenon should be interpreted as veiling of a hue component by the whiteness/ blackness code, $f_{3}$. A rather direct extension of our model which is capable of describing hue veiling by whiteness is given by

$$
H_{i}=c B\left(f_{i} / \sum_{i}^{3} w_{i} f_{i}\right)
$$

In Equation 4, the $\mathrm{w}_{\mathrm{i}}$ are scalars which determine the relative contribution of each $f_{i}$ to the denominator of the equation. That our model still works quite well for the data from our experiment indicates that $w_{1}$ and $w_{2}$ are approximately equal and large as compared to $w_{3}$. Equation 4 directly corresponds to an experiment that is analogous to the one described above. However, even Equation 4 cannot be considered as a complete model in the sense that we do not have a theory for the brightness estimates in terms of the $f_{i}$, i.e., we cannot specify $h$ in $B=$ $h\left(f_{1}, f_{2}, f_{3}\right)$. Although a specification of $h$ in terms of the $f_{i}$ does not seem to be possible at this moment, some attempts have been made to specify $B$ in terms 

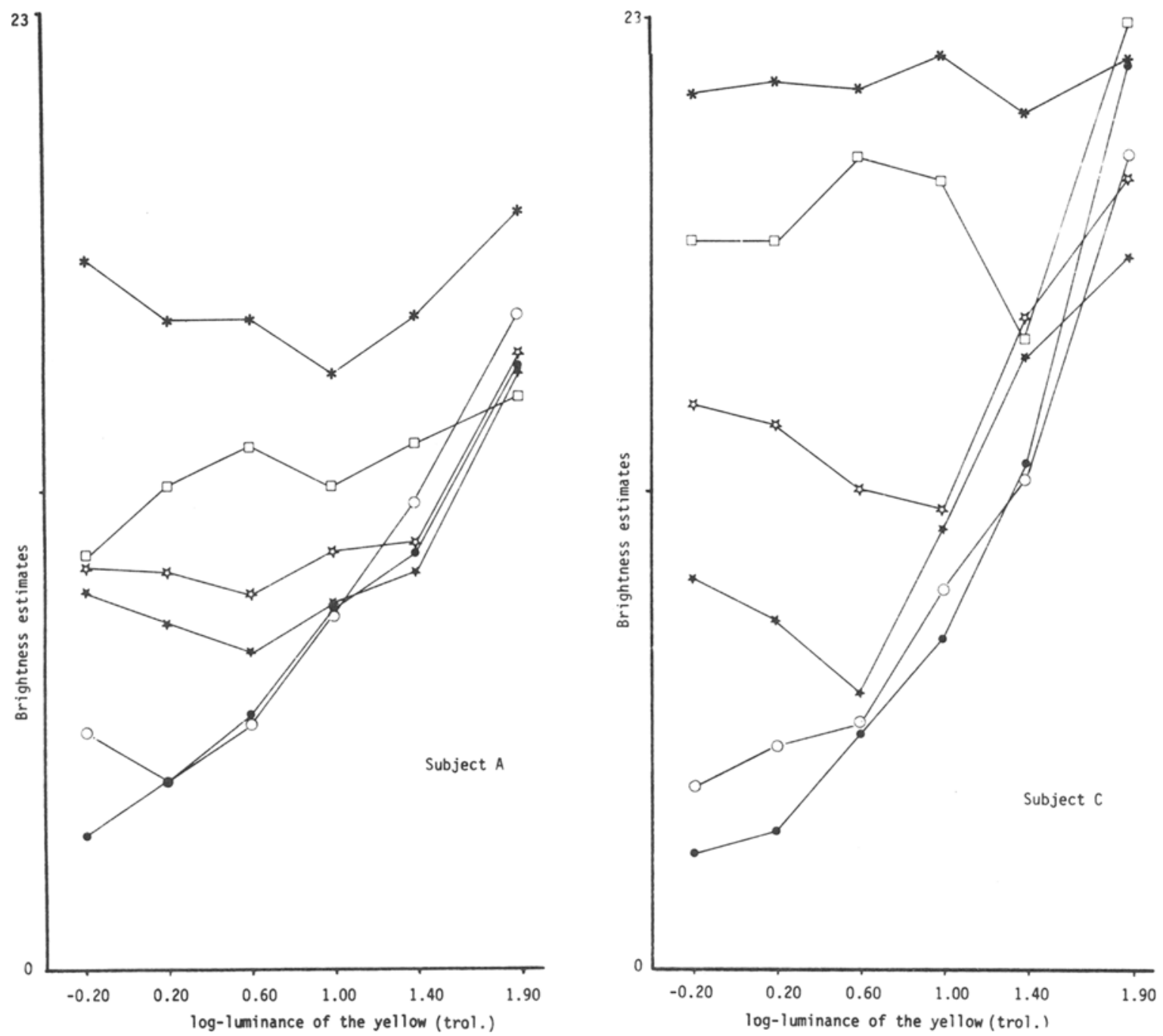

Figure 5. Brightness estimates vs. $\log$ luminance of the yellow stimulus component for different levels of the luminance of the green component $\left(\mathrm{L}_{\mathrm{gr}}\right) . *, \mathrm{~L}_{\mathrm{gr}}=168$ trolands; $\square, \mathrm{L}_{\mathrm{gr}}=53$ trolands; $\star \mathrm{L}_{\mathrm{gr}}=21$ trolands; $\star, \mathrm{L}_{\mathrm{gr}}=8.5$ trolands; $\circ, \mathrm{L}_{\mathrm{gr}}=\mathbf{3 . 5}$ trolands; $\bullet, \mathrm{L}_{\mathrm{gr}}=1.5$ trolands. Each point represents the geometrical mean of five presentations.

of the cancellation energies, notably by Guth and Lodge (1973). In this same article, Guth and Lodge speculate about the possibility of inhibition of the $\mathrm{red} / \mathrm{green}$ system by the yellow/blue system. Our data suggest the existence of such an effect. In Figure 5, the brightness estimates are plotted against the logarithm of the luminance of the yellow component in the stimuli. A simple two-way analysis of variance of the brightness estimates reveals a significant interaction effect of the yellowness and blueness on brightness. From Figure 5, it seems as if the brightness responses are inhibited by the luminance of the yellow as long as this luminance is lower than the luminance of the green component. This indicates that the yellow/biue system inhibits the red/green system for a restricted range of the relative luminances of the yellow and the green. If the hypothesis of $\mathrm{red} / \mathrm{green}$ inhibition by the yellow/blue system is accepted, it should be incorporated in a model for brightness estimates in terms of the nonlinear opponent color codes, $\mathrm{f}_{\mathrm{i}}$.

In order to arrive at a more complete understanding of the veiling phenomenon, it might be interesting to try to quantify veiling itself, for example, by measuring the energy that has to be added to a unique green in a mixture of a unique green and a unique yellow in order to make the mixture contain an equal amount of greenness as the unmixed green did. 


\section{REFERENCE NOTES}

1. Roskam, E. Unidimensional conjoint measurement (UNICON) for multi-faceted designs. Report $74 \mathrm{MA} \mathrm{09,} \mathrm{University}$ of Nijmegen, Department of Mathematical Psychology, 1974.

2. James, F., \& Roos, H. Function minimization. Proceedings of the 1972 CERN Computing and Data Processing School. Report CERN 72-21, 1972.

\section{REFERENCES}

Cicerone, C., Krantz, D., \& Larimer, J. Opponent-process additivity-III: Effect of moderate chromatic adaptation. Vision Research, 1975, 15. 1125-1135.

Guth, S., Donley, N.. \& Marocco, R. On luminance additivity and related topics. Vision Research, 1969, 9, 537-575.

Guth. S., \& LODGE, H. Heterochromatic additivity. foveal spectral sensitivity and a new color model. Journal of the Optical Society of America, 1973, 63, 450-462.

Hurvich, L. M. Two decades of opponent processes. Color 77. International Color Assuciation, 1977.

HuRvich. M. L.. \& Jameson, D. A psychophysical study of white. 1. Neutral adaptation. Journal of the Optical Society of America, 1951, 41, 521-527.

HuRvich, M. L., \& JAMESON, D. An opponent process theory of color vision. Psychological Review, 1957, 64, 384-404.

Jameson, D.. \& HuRvich, M. L. Some quantitative aspects of an opponent colors theory. Journal of the Optical Society of America, 1955, 45, 546-552.

Krantz, D. H. Color measurement and color theory. I. Representation theorem for Grassmann structures. Journal of Mathematical Psychology, 1975, 12, 283-303. (a)

Krantz, D. H. Color measurement and color theory. 11. Opponent colors theory. Journal of Mathematical Psychology. 1975, 12, 304-327. (b)

Krantz, D. H., Luce, D., Suppes, P., \& Tversky, A. Foundaations of measurement. New York: Academic Press, 1971.
Larimer, J., Krantz, D. H., \& Cicerone. C. Opponentprocess additivity-I: Red/green equilibria. Vision Research, 1974. 14. 1127-1139.

Larimer, 3., Krantz, D. H, \& Cicerone, C. Opponentprocess additivity-II: Yellow/blue equilibria and nonlinear models. Vision Research, $1975,15.723 .731$.

LeVelt. W.. Riemersma. J., \& Bunt. A. Binaural additivity of loudness. British Joumal of Mathematical and Statistical Psychology. 1972, 25, 51-68.

RaAIJMAKERS, J., \& DE WEERT, C. Linear and nonlinear opponent color coding. Perception \& Psychophysics, 1975. 18. 474-480.

YAGER, D., \& TAYLOR, E. Experimental measures and a theoretical account of hue scaling as a function of luminance. Perception \& Psychophysics, 1970, 7, 360-364.

\section{NOTES}

1. For the model $Y=\beta X+\varepsilon$, a least squares estimate of $\hat{\beta}$ is provided by $\beta=(\Sigma X Y) /\left(\Sigma X^{2}\right)$, which implies as an estimate of $\sigma_{y . x}^{2}$ the quantity:

$$
s_{y \cdot x}^{2}=\left[\Sigma Y^{2}-(\Sigma X Y) /\left(\Sigma X^{2}\right)\right] /(n-1) .
$$

An estimate of the proportion of the variance of $\mathrm{Y}$, as explained by this model, is the coefficient of determination $d_{y x}$ :

$$
\mathrm{d}_{\mathrm{yx}}=1-\left(\mathrm{s}_{\mathrm{y}, \mathrm{x}}^{2} / \mathrm{s}_{\mathrm{y}}^{2}\right) \text {. }
$$

It is interesting to note that, in general, $d_{y x} \neq d_{y x}$; i.e., $d$ is not a symmetrical measure such as the squared correlation coefficient $r^{2}$. Furthermore, because the lower bound of $d$ is not equal to zero, d cannot always be interpreted as a proportion. That $d$ is nonetheless chosen instead of $r^{2}$ is because $r^{2}$ should be interpreted in terms of explained variance by the model $Y=\beta X+\alpha+\varepsilon$.

(Received for publication March 1, 1977; revision accepted February 14, 1978.) 